\title{
Fractalkine (CX3CL1) and Its Receptor CX3CR1 May Contribute to Increased Angiogenesis in Diabetic Placenta
}

\author{
Dariusz Szukiewicz, ${ }^{1}$ Jan Kochanowski, ${ }^{2}$ Michal Pyzlak, ${ }^{1}$ Grzegorz Szewczyk, \\ Aleksandra Stangret, ${ }^{1}$ and Tarun Kumar Mittal ${ }^{3}$ \\ ${ }^{1}$ Department of General \& Experimental Pathology, Second Faculty of Medicine, Medical University of Warsaw, \\ Ulica Krakowskie Przedmiescie 26/28, 00-928 Warsaw, Poland \\ ${ }^{2}$ Department of Neurology, Second Faculty of Medicine, Medical University of Warsaw, Ulica Ceglowska 80, 01-809 Warsaw, Poland \\ ${ }^{3}$ Department of Obstetrics \& Gynecology, Second Faculty of Medicine, Medical University of Warsaw, \\ Ulica Kondratowicza 8, 03-242 Warsaw, Poland
}

Correspondence should be addressed to Dariusz Szukiewicz; dszukiewicz@hotmail.com

Received 12 March 2013; Revised 12 June 2013; Accepted 26 June 2013

Academic Editor: Janusz Rak

Copyright (C) 2013 Dariusz Szukiewicz et al. This is an open access article distributed under the Creative Commons Attribution License, which permits unrestricted use, distribution, and reproduction in any medium, provided the original work is properly cited.

\begin{abstract}
Chemokine CX3CL1 is unique, possessing the ability to act as a dual agent: chemoattractant and adhesive compound. Acting via its sole receptor CX3CR1, CX3CL1 participates in many processes in human placental tissue, including inflammation and angiogenesis. Strongly upregulated by hypoxia and/or inflammation-induced inflammatory cytokines secretion, CX3CL1 may act locally as a key angiogenic factor. Both clinical observations and histopathological studies of the diabetic placenta have confirmed an increased incidence of hypoxia and inflammatory reactions with defective angiogenesis. In this study we examined comparatively (diabetes class C complicated versus normal pregnancy) the correlation between CX3CL1 content in placental tissue, the mean CX3CR1 expression, and density of the network of placental microvessels. A sandwich enzyme immunoassay was applied for CX3CL1 measurement in placental tissue homogenates, whereas quantitative immunohistochemical techniques were used for the assessment of CX3CR1 expression and the microvascular density. Significant differences have been observed for all analyzed parameters between the groups. The mean concentration of CX3CL1 in diabetes was increased and accompanied by augmented placental microvessel density as well as a higher expression of CX3CR1. In conclusion, we suggest involvement of CX3CL1/CX3CR1 signaling pathway in the pathomechanism of placental microvasculature remodeling in diabetes class C.
\end{abstract}

\section{Introduction}

Augmented immune tolerance during pregnancy prevents onset of inflammatory immune responses that may cause fetal rejection [1]. Specific roles of the cytokine network in the human placenta include local modulation of the balance between pro and anti-inflammatory factors [2]. Thus, together with pregnancy-specific hormones, adequate participation of placental cytokines in immune control is crucial for normal intrauterine growth of the fetus [3]. Since many mediators of inflammation are angiogenic, any disorder associated with the shift in the precise quantitative balance between proinflammatory cytokines and their inhibitors may influence the development of placental vessels [4].
Pathomechanisms of diabetes mellitus during pregnancy include changed oxygen and metabolic pathways, resulting in abnormal placental villous growth and function [5]. It was reported that dysregulation of angiogenic response within diabetic placental tissue may significantly affect fetal wellbeing by an increase in susceptibility to hypoxia and hypoxiaassociated apoptotic triggers [6].

Clinical observations and histopathological studies of the placenta have confirmed an increased incidence of inflammatory reactions in diabetes $[7,8]$. The class $\mathrm{C}$ of diabetes in pregnancy (after White) is the last stage without recognized vascular morphological changes in light microscopy [9] (Table 1). We previously showed that increased density of the villous vascular network in class $\mathrm{C}$ human diabetic 
TABLE 1: Clinical characteristics of the two studied groups: diabetes class C after White (group I) versus normal-course pregnancies (group II).

\begin{tabular}{|c|c|c|}
\hline Parameter & Group I (diabetes class C) & Group II (normal controls) \\
\hline Number of patients/placentae/newborns $(n)$ & $11 / 11 / 11$ & $11 / 11 / 11$ \\
\hline Age of the patients in full years (range; mean; median) & $26-33 ; 29 ; 28$ & $22-33 ; 27 ; 26$ \\
\hline Parity & 0 & 0 \\
\hline Gestational age in days (range; mean; median) & $242-258 ; 250 ; 252$ & $249-257 ; 253 ; 255$ \\
\hline Method of delivery & Cesarean section & Cesarean section \\
\hline 1st minute Apgar's score (range; mean) & $8-10 ; 9.3$ & $9-10 ; 9.7$ \\
\hline Blood pressure during pregnancy & All records within normal range ${ }^{a}$ & All records within normal range ${ }^{\mathrm{a}}$ \\
\hline Proteinuria during pregnancy & Not present $\mathrm{t}^{\mathrm{b}}$ & Not present \\
\hline Liver blood tests (aminotransferases enzymes AST and ALT levels) & Within normal range ${ }^{c}$ & Within normal range $e^{c}$ \\
\hline Smoking during pregnancy & 1 declared $>5$ cigarettes per day & 2 declared $>5$ cigarettes per day \\
\hline Body Mass Index $<21$ or $>35$ & None & None \\
\hline Other identified risk factors & None & None \\
\hline Birth weight in grams (range; mean; median) & $2995-3590 ; 3386 ; 3277$ & $3056-3440 ; 3307 ; 3320$ \\
\hline Newborns gender ( $\mathrm{M}$-male; $\mathrm{F}$-female) & $4 \mathrm{M}+7 \mathrm{~F}$ & $6 \mathrm{M}+5 \mathrm{~F}$ \\
\hline Weight of placenta in grams (range; mean, median) & $425-616 ; 571 ; 563$ & $482-626 ; 554 ; 539$ \\
\hline
\end{tabular}

placenta was correlated with higher histamine and vascular endothelial growth factor (VEGF) concentrations and increased number of placental mast cells $[10,11]$. Since several mastocyte-derived mediators, including histamine, are angiogenic and regulate endothelial cell proliferation and function, degranulation of mast cells may augment local angiogenesis [12]. One explanation for the increase in mast cell number is migration of mast cells in diabetic placental tissue. Considering this, the role of chemotactic cytokines-chemokines in the pathogenesis of diabetes-induced neovascularization-should be suspected [13].

Chemokines form a superfamily of cytokines with the major roles involved in the modulation of immune response and the guidance of migrating leukocytes towards or away from the chemotactic substance (chemoattractant or chemorepellent activities, resp.) [14]. First description of chemokine CX3CL1 (known also as fractalkine or neurotactin) was given in 1997 by Bazan et al. and Pan et al. $[15,16]$. To date, encoded on human chromosome 16 and possessing three amino-acid residues located between the first two cysteine residues in the molecule, CX3CL1 is the lone member of the CX3C (delta) subfamily of chemokines [17]. Unlike other chemokines, CX3CL1 is of nonhaemopoietic origin and exists in a soluble form as chemotactic protein and in a membraneanchored form mainly on endothelial cells as a cell-adhesion molecule. The main roles of the soluble CX3CL1 domain include chemotactic activity for natural killer (NK) cells, T cells, monocytes, and mast cells, but not neutrophils, whereas membrane-anchored form of the chemokine is involved in promotion of leukocyte binding and adhesion. This dual function (chemoattractant and adhesive compound) makes CX3CL1 unique among the known chemokine subfamilies
[18]. Together with some other chemokines (CCL4, CCL7, CCL14) CX3CL1 participates in the processes of implantation, trophoblast invasion into the spiral uterine arteries, placental angiogenesis, response to inflammatory and immunologic factors within the uteroplacental unit, and induction of labor [19-21]. Endothelial cells of the placental vasculature, vascular smooth muscle cells, and amniotic epithelial cells are the main sources of CX3CL1 in the human placenta including the membranes [22-24].

Actions of CX3CL1 are mediated by its sole receptor CX3CR1 (previously known as V28), G $\alpha$ i protein-linked seven-transmembrane receptor [25]. Expression of CX3CR1 was confirmed in endothelial cells, as well as on mast cells and other cell types, including monocytes, NK cells, microglial cells, neurons, and subpopulations of T-lymphocytes [26]. CX3CR1 receptor stimulation leads to the activation of both CX3CL1-dependent and integrin-dependent migrations of cells with augmented adhesion in result of synergistic reactions [27].

Strongly upregulated by hypoxia and/or inflammationinduced inflammatory cytokines secretion, especially tumor necrosis factor alpha $(\mathrm{TNF} \alpha)$, interferon gamma (IFN $\gamma)$, and interleukin-1 beta (IL-1 $\beta$ ), CX3CL1 may act locally as a key angiogenic factor [28]. It was reported that activation of CX3CL1/CX3CR1 signaling pathway induces angiogenesis through two sequential steps: the induction of hypoxia inducible factor 1 alpha (HIF-1 $\alpha$ ) and vascular endothelial growth factor-(VEGF-) A gene expression and the subsequent VEGF-A/vascular endothelial growth factor receptor type 2 (VEGFR2 or KDR) induced angiogenesis [29, 30].

The aim of this study was to examine comparatively (diabetes class $\mathrm{C}$-complicated versus normal pregnancy) the 
correlation between CX3CL1 content in placental tissue, the mean CX3CR1 expression, and density of the network of placental microvessels.

\section{Materials and Methods}

The study was conducted in compliance with international and local laws of human experimentation and was officially approved by local ethics committee, and written consent from the women was obtained for use of their placentae. Strictly speaking, the work has been carried out in accordance with the code of ethics of the World Medical Association (Declaration of Helsinki) for experiments involving humans and the uniform requirements for manuscripts submitted to biomedical journals have been fulfilled.

2.1. Placental Tissue Collection. Eleven placentae obtained from nulliparas after single pregnancies complicated by diabetes class C (group I; the mean gestational age $250 \pm$ 8 days), were compared with 11 placentae obtained from gestationally matched near-term controls (group II; $253 \pm 4$ days). All pregnancies were terminated by cesarean sections due to the fetal interest (group I), including breech presentation (pelvic longitudinal lie of the fetus) in group II. The control of glycemia in all cases was satisfactory; the levels of fraction of glycosylated hemoglobin in all trimesters of diabetic pregnancy were kept within the normal range $(5 \%-$ $7.5 \%)$. The courses of pregnancies in group II were normal, except for a near-term initiation of the contractile activity of the uterus. More detailed clinical characteristics of the two studied groups are given in Table 1 .

Five specimens were excised in a standardized manner from each placenta: three from the region contiguous to the maternal surface (the first one-from the central part, the next two-from peripheral regions of the placental maternal surface) and two specimens from the region contiguous to the fetal surface (the first-from place of umbilical cord insertion, the next-from the peripheral region) (Figure 1). The tissue material obtained by this procedure was subjected either to freezing in carbon dioxide snow for CX3CL1 concentration measurement or fixed in paraffin wax and cut at $5 \mu \mathrm{m}$, before staining procedures with hematoxylin/eosin and immunohistochemistry.

2.2. Measurement of CX3CL1 Content. Concentration of CX3CL1 was estimated in the frozen placental excisions. During handling, the material was kept on ice. Before generating a lysate, the tissue was initially cut into about $1 \mathrm{~mm}^{3}$ cubes by using a razor blade on a glass plate held on ice. In order to perform a gentle cell disruption, the cubes were then transferred into a hand-held Potter S homogenizer. The liquefied tissue was placed in the $1.5 \mathrm{~mL}$ tubes and centrifuged at $13000 \mathrm{rpm}$ for $3 \mathrm{~min}$ at $4^{\circ} \mathrm{C}$. The clear supernatant was used for in vitro quantitative measurement of CX3CL1 in the placental tissue homogenates by a sandwich enzyme immunoassay. Chemokine C-X3-C-Motif Ligand 1 (CX3CL1) BioAssay ELISA Kit (Human; Cat. no. 024096) was applied with detection range $0.156-10 \mathrm{ng} / \mathrm{mL}$ and sensitivity of $0.053 \mathrm{ng} / \mathrm{mL}$. The mean value was calculated for each

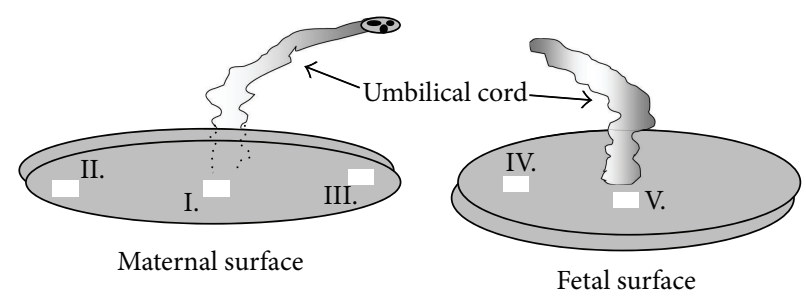

FIGURE 1: Location of samples collected in standardized manner from the maternal surface of the placenta (I-III) and from the fetal surface $(\mathrm{IV}-\mathrm{V})$. The mean weight of the sample: $10.49 \pm 0.89 \mathrm{~g}$.

examined placenta and expressed in $\mathrm{pg} / \mathrm{g}$ of wet tissue weight.

2.3. Immunohistochemistry of CX3CR1. Human placenta paraffin $5 \mu \mathrm{m}$ sections were subjected to the standard immunohistochemical procedures that led to visualization of CX3CR1. Rabbit polyclonal antibody IgG to CX3CR1 (ab8020; Abcam Inc., USA; concentration of $10 \mu \mathrm{g} / \mathrm{mL}$ ) was used as primary and goat anti-rabbit IgG as biotinylated secondary antibody (ab64256; Abcam; $0.5 \% \mathrm{v} / \mathrm{v}$ ). In order to visualize the primary anti-CX3CR1 antibodies, the StreptABComplex/HRP Duet (Dako Cytomation, Glostrup, Denmark) was used, following the protocol recommended by the manufacturer, with $3,3^{\prime}$-diaminobenzidine that served as a chromogen. The respective negative controls for immunostainings were prepared simultaneously by replacement of the polyclonal primary antibody by normal rabbit preimmune IgG diluted with phosphate buffered saline, containing 3\% bovine serum albumin at the same protein concentration as that used for the primary antibody.

2.4. Mean Density of Placental Microvessels. Identification of the vasculature elements in placental sections was performed using endothelial cell marker, rabbit polyclonal antibody antiCD31 (dilution 1:50, ab28364; Abcam Inc., Cambridge, MA, USA). The tissue was incubated with the primary antibody for 30 minutes. Next, a biotinylated goat anti-rabbit antibody was used as the secondary (Abcam).

Using light microscopy with computed morphometry for quantitative analysis (Quantimet 500C+ image analysis workstation provided by Leica, UK), the vascular/extravascular tissular index (V/EVTI) was estimated in calibrated areas of the placental sections. Each preparation (paraffin section) underwent three area analyses repeated by two experienced, independent observers. The single area measured with the picture analyzer amounted to $721320 \mu \mathrm{m}^{2}$ and the total number of preparations 55 per group. The picture analysis procedure consisted in a measurement of the total vascular area. Consequently, the total lumen area of all types of identified vessels was summed up in both groups. With the purpose of a minimizing disruption caused by technical errors, especially unaxial section of the vessel, the lowest value of Ferret's diameter was accepted as the diameter of single lumen. Thus, V/EVTI represents the ratio, which reflects intensity of vascularization and is most closely correlated with the mean density of placental microvessels [31]. 


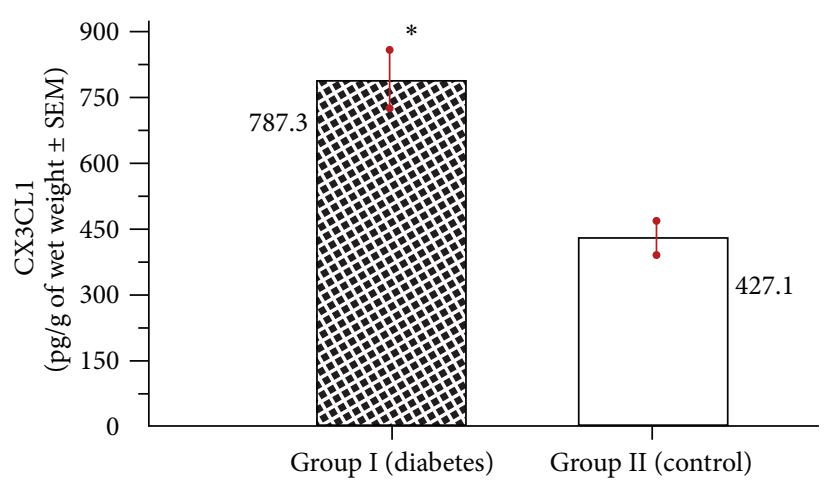

(a)

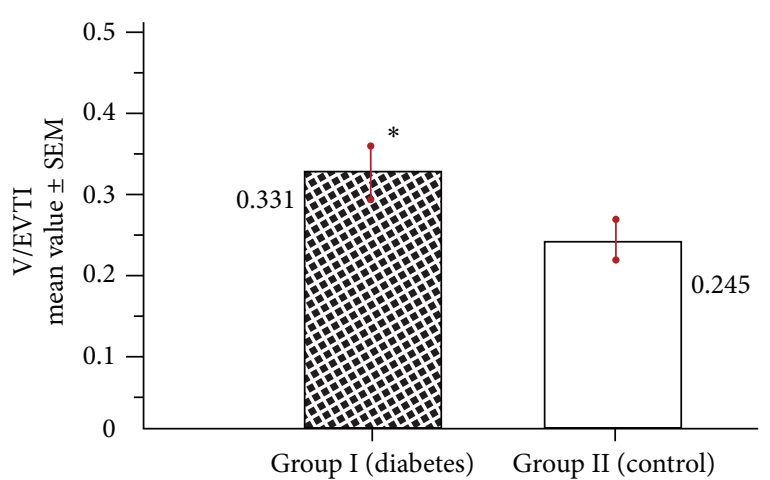

(b)

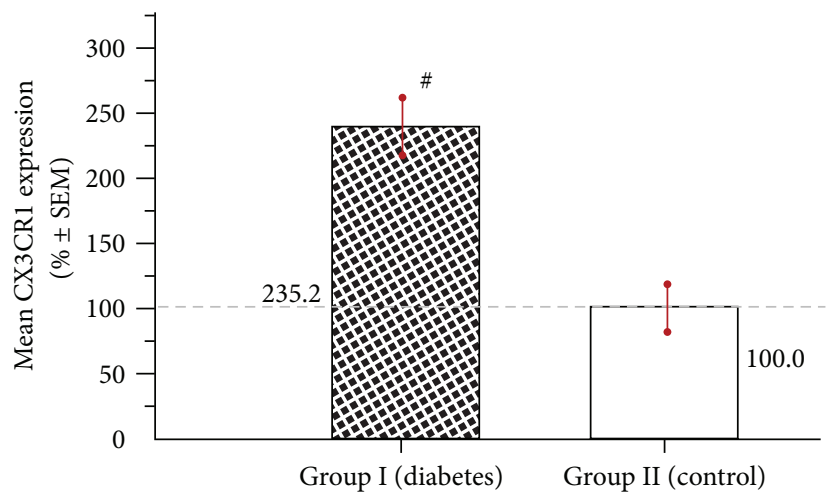

(c)

FIGURE 2: Placental samples collected after diabetes class C (group I) versus specimens obtained after normal pregnancy (group II): (a) the mean concentration of CX3CL1; (b) density of the microvessels estimated using the mean vascular/extravascular tissular index (V/EVTI); (c) the mean percentage expression of CX3CR1. The values obtained for normal pregnancy $(0.016 \pm 0.0014$; abstract numbers \pm SEM) were taken as $100 \%\left({ }^{*} P<0.05 ;{ }^{\#} P<0.01\right)$.

2.5. Expression of CX3CR1. After immunostaining, a quantitative immunohistochemistry based on morphometric software (Quantimet 500C+) was applied for CX3CR1 receptors identification in paraffin $5 \mu \mathrm{m}$ sections of the placental specimens under light microscopy. All morphometric procedures were carried out twice by two independent researchers and the average values uploaded in the result recording tab. Intensity of immunostaining was evaluated using mean colour saturation parameter and thresholding in grey-level histograms. Thus, expression of CX3CR1 corresponded to the total immunostained calibrated area of examined sections, where colour saturation comprises segmentation-separation criteria for objects. Single analysed image area amounted to $138692 \mu \mathrm{m}^{2}$ (magnification $\times 200$ ). In total, 165 visual fields have been analysed (15 visual fields per placenta) in each studied group. To achieve maximum accuracy of measurements, the following factors have been controlled or monitored: averaging of image intake, hue, illumination, luminance, power supply, relation of illumination to quantification of area percentage of positively staining structures, shading correction, and warming up. More detailed description of these morphometric procedures was given previously elsewhere [31, 32]. Assuming that the accuracy of CX3CR1 expression measurement may be significantly affected by the local differences in density of placental microvessels, in both groups we examined comparatively vascular densitymatched samples with the tolerated range of discrepancy $\pm 5 \%$ [33]. Morphometric results comprising $90 \%$ confidence intervals were reported as mean percentage values \pm SEM.

2.6. Statistical Analysis. Statistical analyses were performed using Statistica 8.0 software (Stat-Soft, Poland). MannWhitney's $U$ test was applied. The results were expressed as means \pm SEM or mean percentage values \pm SEM. Differences between group I (diabetes class C) and group II (normalcourse pregnancy) were deemed statistically significant if $P<$ 0.05 .

\section{Results}

The results pertaining to CX3CL1 content in placental tissue, the mean density of microvessels, and CX3CR1 expression are summarized in Figures 2(a), 2(b), and 2(c), respectively. Significant differences have been observed for all analyzed parameters between the groups.

The mean concentration of CX3CL1 in placental cuts from the diabetic group (group I) was increased compared to controls (group II) and amounted to $787.3 \pm 70.2 \mathrm{pg} / \mathrm{g}$ of wet weight \pm SEM versus $427.1 \pm 37.4$ (Figure $2(\mathrm{a})$ ). There were no significant differences in CX3CL1 levels between the 


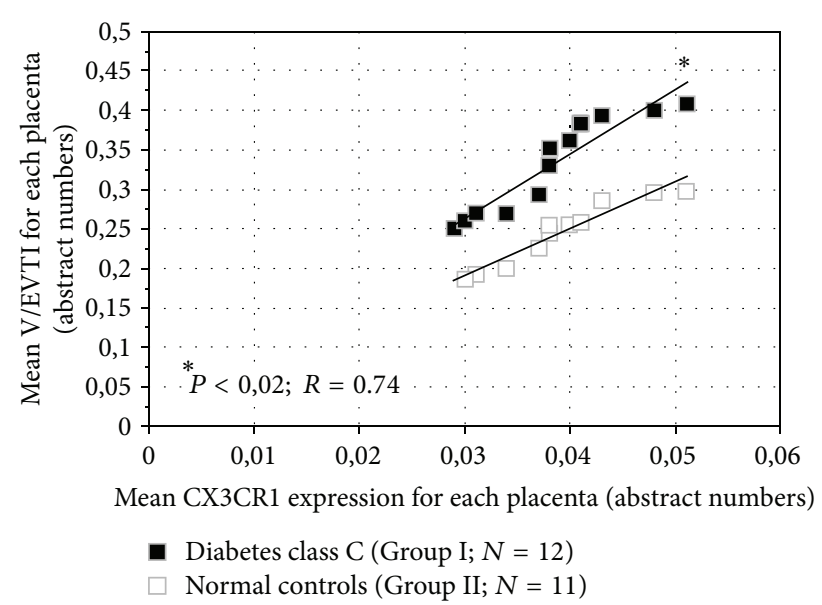

FIGURE 3: The mean values of V/EVTI versus the mean CX3CR1 expressions: diabetic and normal placentae, comparatively.

specimens collected from maternal and fetal surface of the placenta within the group.

The mean V/EVTI value (absolute number \pm SEM) in placental tissue obtained after diabetes class C-complicated pregnancies was increased $(P<0.05)$, amounting to $0.331 \pm$ 0.037 versus $0.245 \pm 0.025$ in normal controls (Figure 2(b)). These mean values of V/EVTI were evaluated totally for each group, using the partial data gained from all collected placental samples. However, we observed in both groups that in the samples obtained from the maternal surface, the mean density of microvessels expressed as V/EVTI was significantly higher $(P<0.05)$ compared to the mean V/EVTI calculated for the specimens of placental tissue obtained from the fetal surface (the data not shown on the chart).

Evaluation of the relationship between the mean CX3CR1 expression and the mean V/EVTI revealed strong positive correlation and significant differences between the groups (Figure 3). The higher expression of CX3CR1 in diabetes corresponded to the augmentation of the placental vascularization, as assessed by V/EVTI.

Immunohistochemical technique used for identification of CX3CR1 revealed that this receptor is predominantly located in placental endothelial cells (Figure 4). The mean percentage value of CX3CR1 expression estimated in the vascular density-matched samples was remarkably higher $(P<0.01)$ in diabetes and reached $235.2 \pm 24.4(\%, \pm$ SEM $)$ of the reference value established in group II (Figure 2(c)).

\section{Discussion}

The growth, maintenance and functioning of placental microvessels may be affected by the majority of the pathological changes in maternal/fetal hemodynamics and by alterations in blood composition or content [34]. Different authors working independently have reported that the induction of the pro-inflammatory milieu is inherently involved in the pathophysiology of diabetes mellitus, including diabetic placenta $[7,35]$. In such an environment, frequent episodes of local hypoxia and transient hyperglycemia (even in well-controlled cases) are observed and are associated with elevated levels of free oxygen radicals, advanced glycation end products (AGEs), and some proinflammatory cytokines, especially those with angiogenic properties $[6,35]$. However, it has also been reported that placental expression of the inflammatory cytokines in response to oxidative stress is significantly reduced in gestational diabetes mellitus [36]. This study did not involve women with diabetes class $\mathrm{C}$, and CX3CL1 concentrations were not examined.

Secondary to hypoxia, increases in the local levels of TNF $\alpha$, IFN $\gamma$, and IL- $1 \beta$ potentiate the angiogenic action of CX3CL1 that is simply correlated with an increase in the concentration of this chemokine $[28,29,37]$. CX3CL1 stimulates ex vivo and in vivo angiogenesis in a dose- and timedependent manner and acts on endothelial cells even more strongly than the well-known angiogenic factor VEGF [29]. It has been reported that CX3CL1 (100 nM) induced endothelial cells to form capillary tubes in synthetic matrix with at least the same efficiency as the angiogenic mediators bFGF $(60 \mathrm{nM})$ and VEGF $(100 \mathrm{nM})$ [38]. Based on the data that are available to date, there is no clear standpoint on the roles of VEGF and its receptor VEGFR-2 (KDR/Flt-1) in CX3CL1mediated angiogenesis. Some authors have reported that the activation of CX3CL1/CX3CR1 by vascular endothelial cells induces angiogenesis through VEGF-A/KDR. Increased VEGF production can be achieved through the pathways involving hypoxia-inducible factor-1 alpha (HIF-1 $\alpha$ ) and p42/44 mitogen-activated protein (MAP) kinase [29]. In our previous study of the diabetic placenta, we found that higher VEGF expression levels were positively correlated with increased microvascular density [11]. However, the results of another study indicate that the mechanism of CX3CL1 expression and its angiogenic effects differ from those of VEGF. Whereas hypoxia strongly induces VEGF expression, an ischemic environment actually inhibits the expression of CX3CL1. This finding may suggest that hypoxia promotes VEGF-mediated angiogenesis, while inflammation involves CX3CL1-related new vessel formation [39]. Considering this, our findings are rather correlative and not mechanistic in nature. The results provide no formal evidence for the causal involvement of the CX3CL1/CX3CR1 system in altered placental vascularity, especially because we have previously reported changes in the placental VEGF expression [11].

Studies of the molecular mechanism by which CX3CL1 regulates angiogenesis in human umbilical vein endothelial cells (HUVEC) showed that CX3CL1 did not increase the levels of VEGF mRNA. These results indicate that CX3CL1 may act as a direct angiogenic modulator in endothelial cells without inducing VEGF expression. It is likely that the angiogenic activities of CX3CL1 and VEGF are independent of one another but utilize the Raf1/MEK/ERK kinase cascade and $\mathrm{P} 13 \mathrm{~K} / \mathrm{Akt} / \mathrm{eNOS}$ activation as common mediators in their angiogenic signaling pathways. In other words, it was proposed recently that CX3CL1, acting directly on angiogenesis via CX3CR1, may activate the two previously mentioned distinct signaling pathways [40].

All of the characteristic steps in the complex process of new vessel formation are augmented after CX3CL1 treatment, 


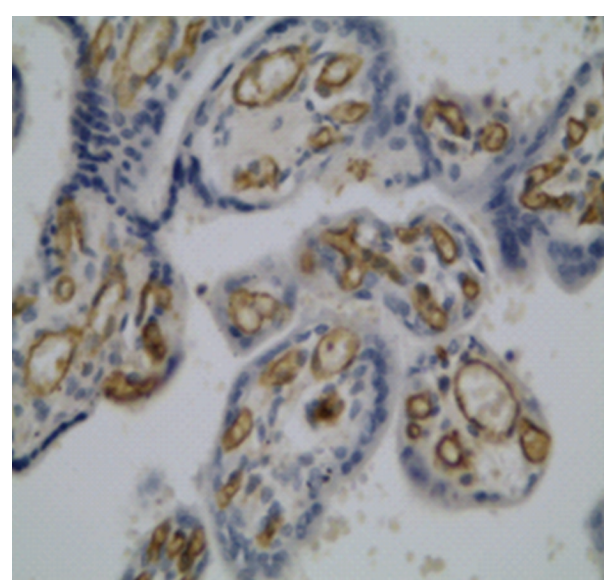

(a)

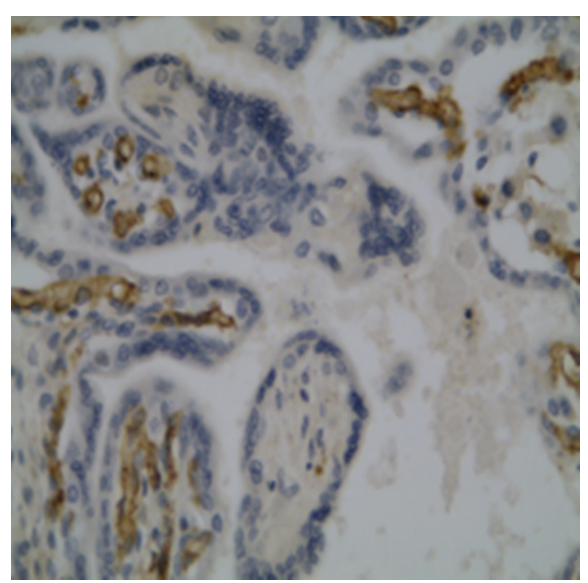

(b)

FIGURE 4: Immunohistochemical localization of the receptor CX3CR1 in the placental tissue at 400x magnification: (a) diabetes class C (group I); (b) normal controls (group II).

including endothelial cell proliferation, migration, and tubelike structure formation $[30,41]$.

The statistically significant upregulation of CX3CR1 expression documented in diabetic endothelial cells should also be considered, particularly because of the existence of an autoregulatory mechanism between CX3CL1 and CX3CR1 [42]. A form of autoregulation between CX3CR1 and CX3CL1 via the autocrine loop (CX3CR1/CX3CL1 axis) was proposed by independent authors with respect to many cell types, including endothelial cells [43-45]. So far, however, there is no convincing scientific evidence that the same human endothelial cells possess the ability to produce CX3CL1 and simultaneously express CX3CR1. However, because CX3CR1 is the only known receptor for CX3CL1, it is possible that CX3CL1induced endothelial cell migration and capillary tube formation was mediated through the interaction between CX3CL1 and its endothelial receptor CX3CR1 in an autocrine manner [38].

It is worth noting that the CX3CR1 signal documented in Figure 4 and considered to be associated with endothelial cells may also come from pericytes. A double-staining technique or high-resolution, high-power imaging should be implemented in future studies to elucidate this issue.

An excess of CX3CL1 and other inflammatory mediators may be responsible for augmented angiogenesis within the placental unit. According to the results of other studies, the regulation of CX3CL1 function may be maintained on both protein and gene expression levels. Increased expression of matrix metalloproteinases (MMPs) and disintegrin metalloproteinases (ADAMs, including ADAM17/TACE) or sheddases have been reported in the diabetic placenta [46, 47]. These increases may lead to an increase in the concentration of the soluble form of CX3CL1, which is derived from its membrane form.

In contrast, the proinflammatory background of diabetes, which includes increased placental levels of TNF $\alpha, \operatorname{IFN} \gamma$, and IL-1 $\beta$, promotes enhanced CX3CL1 gene expression [48].
Further studies are needed to determine which mechanism of CX3CL1 regulation predominates in diabetes class C.

The classic example of a diabetic complication with increased defective angiogenesis mediated by CX3CL1 with the inflammatory background is proliferative retinopathy [38, 49].

Both in this study and in previous reports, we have provided evidence of altered vascularization in the diabetic placenta. The results of the present investigation strongly support the hypothesis that CX3CL1-related pathways is involved in the pathomechanism of placental angiogenesis in diabetes. Increased V/EVTI values are consistent with our previous approach to evaluating the density of placental microvessels in diabetes class $\mathrm{C}$ with respect to histamine concentration and mast cell number $[10,11]$.

Chemotaxis assays performed in vitro by other researchers have demonstrated that increased CX3CL1 levels initiate and augment the migration of specific CX3CR1-positive subpopulations of inflammatory cells [50]. For example, we previously observed both an increase in the number of mast cells in the diabetic placenta and changes in their heterogeneity, namely, a shift of the quantitative balance between tryptase-positive and tryptase/chymase-positive cells [51]. This shift may influence the placental cytokine network profile in diabetes, possibly including an increase in the local CX3CL1 concentration. Considering the dual role of CX3CL1, mechanisms that influence placental angiogenesis and are related to the direct action of CX3CL1 and the chemoattractant properties of CX3CL1 should be considered. Interestingly, despite the significant expression of CX3CR1 by human mast cells, CX3CL1 does not directly produce mast cell degranulation [52]. Thus, CX3CL1 should not be simply linked with mast cell-mediated angiogenesis [12].

It should be clearly stated that altered vascular patterns are not necessarily a result of angiogenesis and could represent a form of vascular remodeling rather than overt capillary growth. This phenomenon is illustrated by the 
larger, rather than more numerous vessels depicted in Figure 4. The algorithm used to enumerate vascular changes does not permit the capturing of microvessels [53].

In conclusion, it is very likely that increased CX3CL1 concentration in diabetes class $\mathrm{C}$, together with the upregulation of its specific and sole receptor, CX3CR1, are involved in the pathomechanism of placental microvasculature remodeling. Further studies are needed to elucidate the rationale for anti-CX3CL1 or anti-CX3CR1 therapies during diabetic pregnancy.

\section{Conflict of Interests}

The authors report no conflict of interests related to this study or the findings specified in this paper.

\section{References}

[1] B. M. Kumpel and M. S. Manoussaka, "Placental immunology and maternal alloimmune responses," Vox Sanguinis, vol. 102, no. 1, pp. 2-12, 2012.

[2] S. Hauguel-de Mouzon and M. Guerre-Millo, "The placenta cytokine network and inflammatory signals," Placenta, vol. 27, no. 8, pp. 794-798, 2006.

[3] D. W. Park and K. M. Yang, "Hormonal regulation of uterine chemokines and immune cells," Clinical and Experimental Reproductive Medicine, vol. 38, no. 4, pp. 179-185, 2011.

[4] D. Ribatti and E. Crivellato, "Immune cells and angiogenesis," Journal of Cellular and Molecular Medicine, vol. 13, no. 9, pp. 2822-2833, 2009.

[5] G. Daskalakis, S. Marinopoulos, V. Krielesi et al., "Placental pathology in women with gestational diabetes," Acta Obstetricia et Gynecologica Scandinavica, vol. 87, no. 4, pp. 403-407, 2008.

[6] L. Leach, A. Taylor, and F. Sciota, "Vascular dysfunction in the diabetic placenta: causes and consequences," Journal of Anatomy, vol. 215, no. 1, pp. 69-76, 2009.

[7] A. Jawerbaum and E. González, "Diabetic pregnancies: the challenge of developing in a pro-inflammatory environment," Current Medicinal Chemistry, vol. 13, no. 18, pp. 2127-2138, 2006.

[8] G. Desoye and S. Hauguel-De Mouzon, "The human placenta in gestational diabetes mellitus: the insulin and cytokine network," Diabetes Care, vol. 30, no. 2, pp. S120-S126, 2007.

[9] P. White, "Classification of obstetric diabetes," American Journal of Obstetrics and Gynecology, vol. 130, no. 2, pp. 228-230, 1978.

[10] D. Szukiewicz, A. Szukiewicz, D. Maslinska, and M. W. Markowski, "Placental mast cells (MC) and histamine (HA) in pregnancy complicated by diabetes class $\mathrm{C}$-relation to the development of villous microvessels," Placenta, vol. 20, supplement 1, pp. 503-510, 1999.

[11] D. Szukiewicz, A. Szukiewicz, D. Maslinska, G. Szewczyk, and M. Watroba, "Mast cell-derived vascular endothelial growth factor (VEGF) and microvascular density in diabetic placentae," Inflammation Research, vol. 52, no. 1, pp. S09-S10, 2003.

[12] K. Norrby, "Mast cells and angiogenesis: review article," APMIS, vol. 110, no. 5, pp. 355-371, 2002.

[13] J. Zeng, K. Xie, H. Wu, B. Zhang, and C. Huang, "Identification and functional study of cytokines and chemokines involved in tumorigenesis," Combinatorial Chemistry and High Throughput Screening, vol. 15, no. 3, pp. 276-285, 2012.
[14] A. Zlotnik and O. Yoshie, "The chemokine superfamily revisited," Immunity, vol. 36, no. 5, pp. 705-716, 2012.

[15] J. F. Bazan, K. B. Bacon, G. Hardiman et al., "A new class of membrane-bound chemokine with a $\mathrm{CX}_{3} \mathrm{C}$ motif," Nature, vol. 385, no. 6617, pp. 640-644, 1997.

[16] Y. Pan, C. Lloyd, H. Zhou et al., "Neurotactin, a membraneanchored chemokine upregulated in brain inflammation," Nature, vol. 389, no. 6646, article 100, 1997.

[17] H. Nomiyama, T. Imai, J. Kusuda, R. Miura, D. F. Callen, and O. Yoshie, "Human chemokines fractalkine (SCYD1), MDC (SCYA22) and TARC (SCYA17) are clustered on chromosome 16q13," Cytogenetics and Cell Genetics, vol. 81, no. 1, pp. 10-11, 1998.

[18] B. A. Jones, M. Beamer, and S. Ahmed, "Fractalkine/CX3CL1: a potential new target for inflammatory diseases," Molecular Interventions, vol. 10, no. 5, pp. 263-270, 2010.

[19] E. Dimitriadis, G. Nie, N. J. Hannan, P. Paiva, and L. A. Salamonsen, "Local regulation of implantation at the human fetal-maternal interface," International Journal of Developmental Biology, vol. 54, no. 2-3, pp. 313-322, 2010.

[20] J. M. Bowen, L. Chamley, M. D. Mitchell, and J. A. Keelan, "Cytokines of the placenta and extra-placental membranes: biosynthesis, secretion and roles in establishment of pregnancy in women," Placenta, vol. 23, no. 4, pp. 239-256, 2002.

[21] A. Taki, M. Abe, M. Komaki et al., "Expression of angiogenesisrelated factors and inflammatory cytokines in placenta and umbilical vessels in pregnancies with preeclampsia and chorioamnionitis/funisitis," Congenital Anomalies, vol. 52, no. 2, pp. 97-103, 2012.

[22] K. Shimoya, Q. Zhang, K. Tenma et al., "Fractalkine (FRK) levels in amniotic fluid and its production during pregnancy," Molecular Human Reproduction, vol. 9, no. 2, pp. 97-101, 2003.

[23] H. Umehara, E. T. Bloom, T. Okazaki, Y. Nagano, O. Yoshie, and T. Imai, "Fractalkine in vascular biology: from basic research to clinical disease," Arteriosclerosis, Thrombosis, and Vascular Biology, vol. 24, no. 1, pp. 34-40, 2004.

[24] J. E. Cartwright, R. J. Keogh, and M. C. T. V. Patot, "Hypoxia and placental remodelling," Advances in Experimental Medicine and Biology, vol. 618, pp. 113-126, 2007.

[25] K.-W. Kim, A. Vallon-Eberhard, E. Zigmond et al., "In vivo structure/function and expression analysis of the $\mathrm{CX}_{3} \mathrm{C}$ chemokine fractalkine," Blood, vol. 118, no. 22, pp. e156-e167, 2011.

[26] A. K. Clark, A. A. Staniland, and M. Malcangio, "Fractalkine/ CX3CR1 signalling in chronic pain and inflammation," Current Pharmaceutical Biotechnology, vol. 12, no. 10, pp. 1707-1714, 2011.

[27] J. G. D’Haese, I. E. Demir, H. Friess, and G. O. Ceyhan, "Fractalkine/CX3CR1: why a single chemokine-receptor duo bears a major and unique therapeutic potential," Expert Opinion on Therapeutic Targets, vol. 14, no. 2, pp. 207-219, 2010.

[28] T. Imaizumi, H. Yoshida, and K. Satoh, "Regulation of CX3CL1/fractalkine expression in endothelial cells," Journal of Atherosclerosis and Thrombosis, vol. 11, no. 1, pp. 15-21, 2004.

[29] J. Ryu, C.-W. Lee, K.-H. Hong et al., "Activation of fractalkine/ CX3CR1 by vascular endothelial cells induces angiogenesis through VEGF-A/KDR and reverses hindlimb ischaemia," Cardiovascular Research, vol. 78, no. 2, pp. 333-340, 2008.

[30] B. Mehrad, M. P. Keane, and R. M. Strieter, "Chemokines as mediators of angiogenesis," Thrombosis and Haemostasis, vol. 97, no. 5, pp. 755-762, 2007.

[31] D. Szukiewicz, M. Gujski, D. Maslinska, G. Szewczyk, M. Bachanek, and S. Maslinski, "Mast cell-derived VEGF and 
VEGF receptor type 1, 2, and 3 expression in human term trophoblast culture-influence of hypoxia," Inflammation Research, vol. 54, no. 1, pp. S82-S83, 2005.

[32] B. Huppertz, E. Abe, P. Murthi, T. Nagamatsu, D. Szukiewicz, and C. Salafia, "Placental angiogenesis, maternal and fetal vessels-a workshop report," Placenta, vol. 28, pp. S94-S96, 2007.

[33] K. J. Brown, S. F. Maynes, A. Bezos, D. J. Maguire, M. D. Ford, and C. R. Parish, "A novel in vitro assay for human angiogenesis," Laboratory Investigation, vol. 75, no. 4, pp. 539-555, 1996.

[34] L. P. Reynolds, M. E. Biondini, P. P. Borowicz et al., "Functional significance of developmental changes in placental microvascular architecture: the sheep as a model," Endothelium, vol. 12, no. 1-2, pp. 11-19, 2005.

[35] T. M. Mayhew, D. S. Charnock-Jones, and P. Kaufmann, "Aspects of human fetoplacental vasculogenesis and angiogenesis. III. Changes in complicated pregnancies," Placenta, vol. 25, no. 2-3, pp. 127-139, 2004.

[36] M. Lappas, A. Mittion, and M. Permezel, "In response to oxidative stress, the expression of inflammatory cytokines and antioxidant enzymes are impaired in placenta, but not adipose tissue, of women with gestational diabetes," Journal of Endocrinology, vol. 204, no. 1, pp. 75-84, 2010, Erratum in: Journal of Endocrinology, vol. 204, no. 2, p. 221, 2010.

[37] J. Zhang, H. Hu, N. L. Palma et al., "Hypoxia-induced endothelial CX3CL1 triggers lung smooth muscle cell phenotypic switching and proliferative expansion," American Journal of Physiology, vol. 303, no. 10, pp. L912-L922, 2012.

[38] J.-J. You, C.-H. Yang, J.-S. Huang, M.-S. Chen, and C.-M. Yang, "Fractalkine, a CX3C chemokine, as a mediator of ocular angiogenesis," Investigative Ophthalmology and Visual Science, vol. 48, no. 11, pp. 5290-5298, 2007.

[39] K. Yamashita, T. Imaizumi, M. Hatakeyama et al., "Effect of hypoxia on the expression of fractalkine in human endothelial cells," Tohoku Journal of Experimental Medicine, vol. 200, no. 4, pp. 187-194, 2003.

[40] S.-J. Lee, S. Namkoong, Y.-M. Kim et al., "Fractalkine stimulates angiogenesis by activating the Raf-1/MEK/ERK- and PI3K/Akt/eNOS-dependent signal pathways," American Journal of Physiology, vol. 291, no. 6, pp. H2836-H2846, 2006.

[41] M. V. Volin, J. M. Woods, M. A. Amin, M. A. Connors, L. A. Harlow, and A. E. Koch, "Fractalkine: a novel angiogenic chemokine in rheumatoid arthritis," American Journal of Pathology, vol. 159, no. 4, pp. 1521-1530, 2001.

[42] N. Mizutani, T. Sakurai, T. Shibata et al., "Dose-dependent differential regulation of cytokine secretion from macrophages by fractalkine," Journal of Immunology, vol. 179, no. 11, pp. 74787487, 2007.

[43] S. Morimura, M. Sugaya, and S. Sato, "Interaction between CX3CL1 and CX3CR1 Regulates Vasculitis Induced by Immune Complex Deposition," American Journal of Pathology, vol. 182, no. 5, pp. 1640-1647, 2013.

[44] G. Sciumé, A. Soriani, M. Piccoli, L. Frati, A. Santoni, and G. Bernardini, "CX3CR1/CX3CL1 axis negatively controls glioma cell invasion and is modulated by transforming growth factorbetal," Neuro-Oncology, vol. 12, no. 7, pp. 701-710, 2010.

[45] B. Chandrasekar, S. Mummidi, R. P. Perla et al., "Fractalkine (CX3CL1) stimulated by nuclear factor $\kappa \mathrm{B}$ (NF- $\kappa \mathrm{B}$ )-dependent inflammatory signals induces aortic smooth muscle cell proliferation through an autocrine pathway," Biochemical Journal, vol. 373, no. 2, pp. 547-558, 2003.
[46] M. C. Pustovrh, A. Jawerbaum, E. Capobianco, V. White, J. J. López-Costa, and E. González, "Increased matrix metalloproteinases 2 and 9 in placenta of diabetic rats at midgestation," Placenta, vol. 26, no. 4, pp. 339-348, 2005.

[47] S. Kuc, E. J. Wortelboer, M. P. H. Koster, H. W. De Valk, P. C. J. I. Schielen, and G. H. A. Visser, "Prediction of macrosomia at birth in type-1 and 2 diabetic pregnancies with biomarkers of early placentation," An International Journal of Obstetrics and Gynaecology, vol. 118, no. 6, pp. 748-754, 2011.

[48] Y. Mitamura, C. Harada, and T. Harada, "Role of cytokines and trophic factors in the pathogenesis of diabetic retinopathy," Current Diabetes Reviews, vol. 1, no. 1, pp. 73-81, 2005.

[49] J. T. Durham and I. M. Herman, "Microvascular modifications in diabetic retinopathy," Current Diabetes Reports, vol. 11, no. 4, pp. 253-264, 2011.

[50] Y. Ito, H. Kawachi, Y. Morioka et al., "Fractalkine expression and the recruitment of CX3CR1+ cells in the prolonged mesangial proliferative glomerulonephritis," Kidney International, vol. 61, no. 6, pp. 2044-2057, 2002.

[51] D. Szukiewicz, D. Maslinska, P. Poppe, M. Gujski, and A. Tomecki, "Placental mast cell heterogeneity in pregnancy complicated by diabetes class C," Inflammation Research, vol. 49, no. 1, pp. S33-S34, 2000.

[52] E. J. Papadopoulos, D. J. Fitzhugh, C. Tkaczyk et al., "Mast cells migrate, but do not degranulate, in response to fractalkine, a membrane-bound chemokine expressed constitutively in diverse cells of the skin," European Journal of Immunology, vol. 30, no. 8, pp. 2355-2361, 2000.

[53] P. Carmeliet and R. K. Jain, "Principles and mechanisms of vessel normalization for cancer and other angiogenic diseases," $\mathrm{Na}$ ture Reviews Drug Discovery, vol. 10, no. 6, pp. 417-427, 2011. 


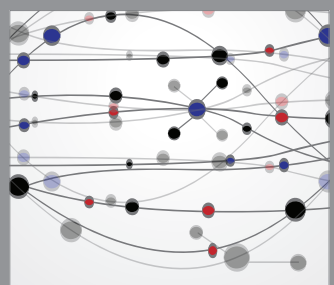

The Scientific World Journal
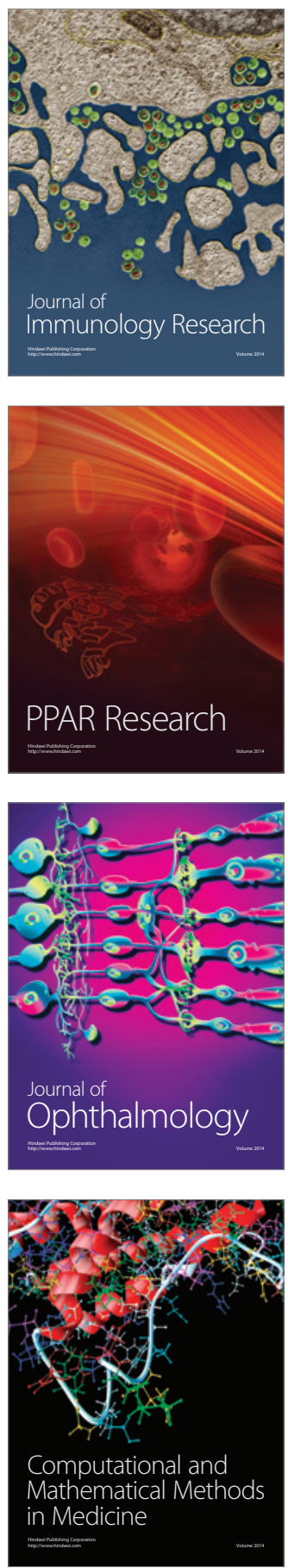

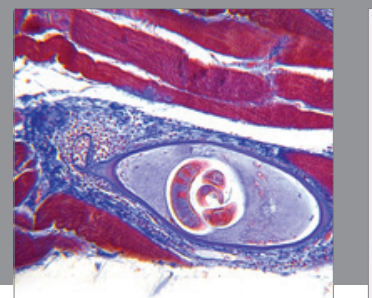

Gastroenterology

Research and Practice
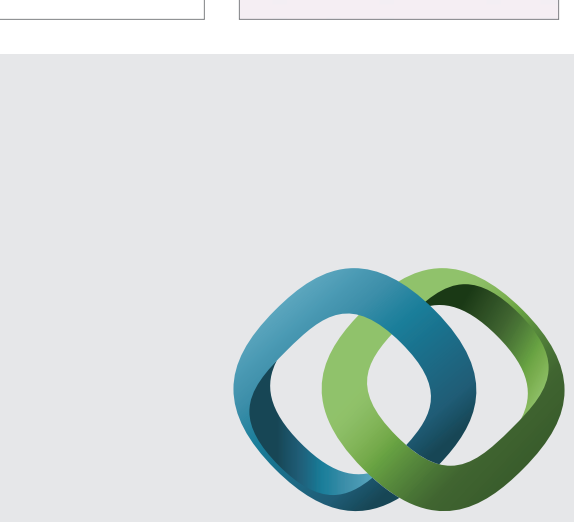

\section{Hindawi}

Submit your manuscripts at

http://www.hindawi.com
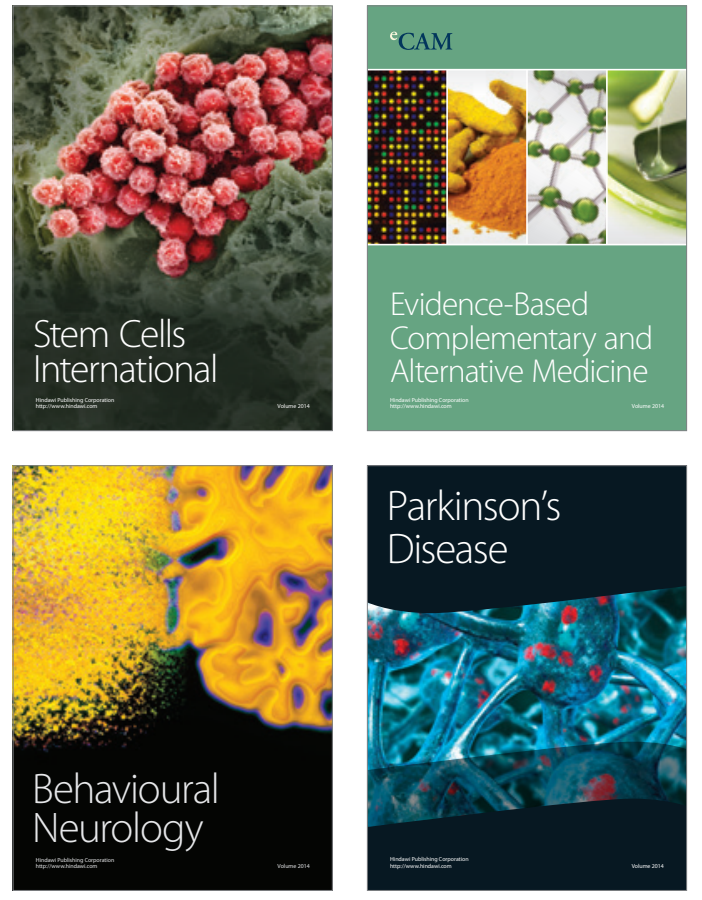
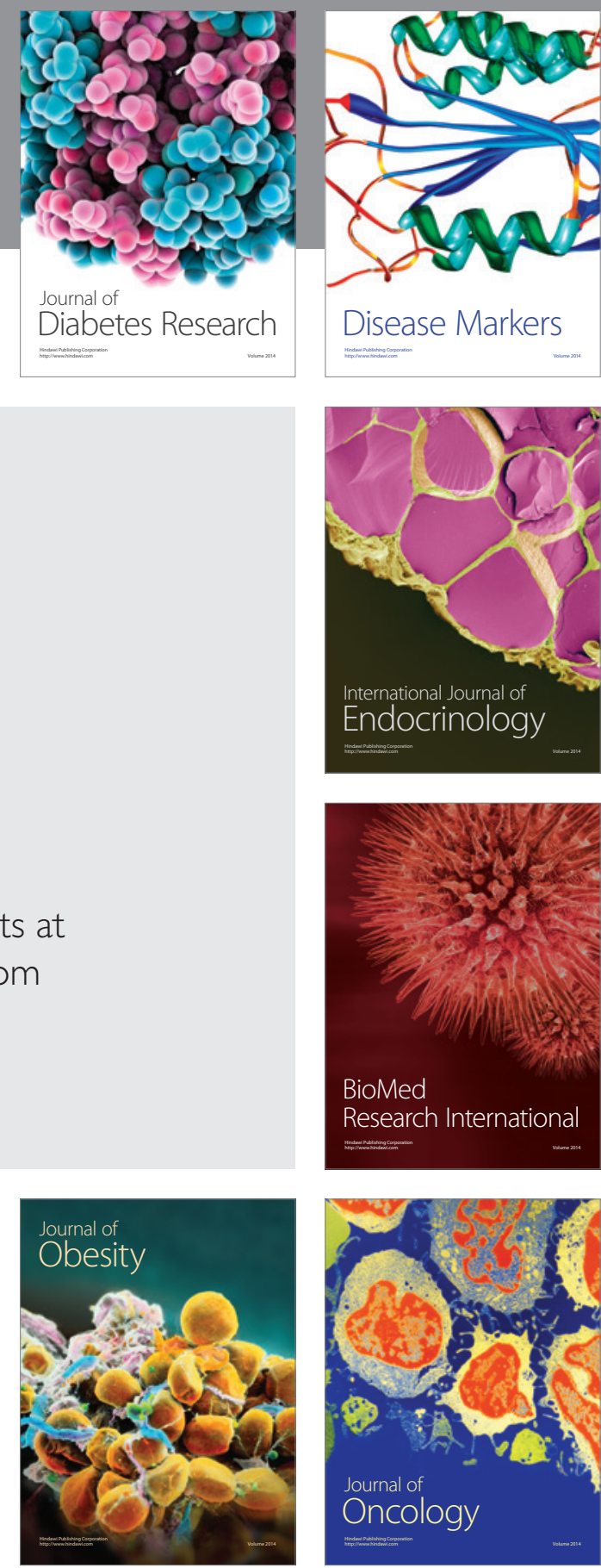

Disease Markers
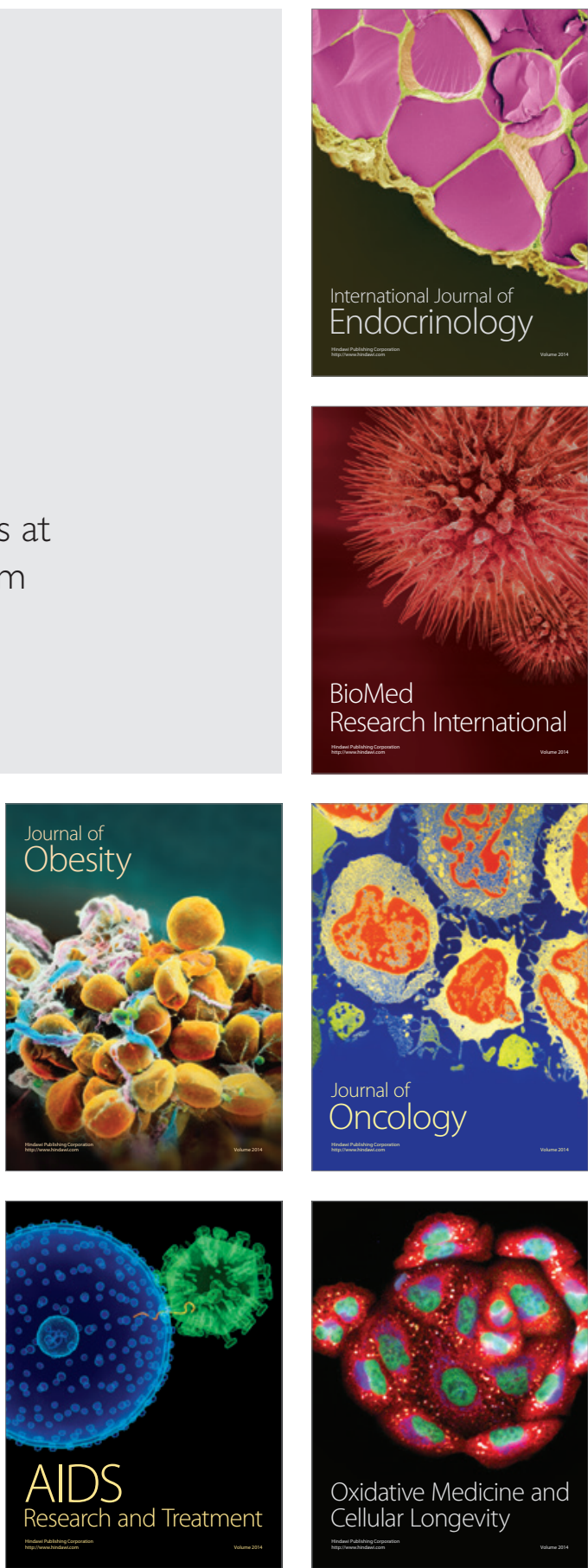